\title{
Herramienta de evaluación de la Propiedad Intelectual para proyectos. Estudio de caso en proyectos de Ciencia, Tecnología e Innovación
}

\author{
Maytee Martínez Domínguez \\ Delegación Territorial de Ciencia, Tecnología y Medio Ambiente (CITMA). \\ Pinar del Río, Cuba \\ maite@citma.vega.inf.cu
}

\section{Isabel García Rodríguez}

Centro de Estudios de Gestión de Ciencias e Innovación. Instituto Superior de

Tecnologías y Ciencias Aplicadas (INSTEC). Ministerio de Educación Superior (MES),

Cuba

igarcia@instec.cu

\section{Elena Figueroa Cabrera}

Departamento Informática, Facultad de Ciencias Técnicas. Universidad de Pinar del Río

Hermanos Saiz Montes de Oca, Cuba

elena@upr.edu.cu

\section{Adriana Fernández Sánchez}

Universidad de Pinar del Río Hermanos Saiz Montes de Oca, Cuba

andry6595@nauta.cu

Cita sugerida: Martínez Domínguez, M., García Rodríguez, I., Figueroa Cabrera, E. y Fernández Sánchez, A. (2018). Herramienta de evaluación de la Propiedad Intelectual para proyectos. Estudio de caso en proyectos de Ciencia, Tecnología e Innovación. Palabra Clave (La Plata), 8(1), e061. https://doi.org/10.24215/18539912e061

Recibido: 6 de diciembre de 2017 - Aceptado: 28 de agosto de 2018 - Publicado: 31 de octubre de 2018

(c) (1) (2) (2) Esta obra está bajo licencia Creative Commons Atribución-NoComercial-CompartirIgual 4.0 Internacional BY No $\mathrm{sA} h \mathrm{http}: / /$ creativecommons.org/licenses/by-nc-sa/4.0/deed.es_AR 


\section{Herramienta de evaluación de la Propiedad Intelectual para proyectos. Estudio de caso en proyectos de Ciencia, Tecnología e Innovación}

Tool of evaluation of the Intellectual Property for projects. Study of case in projects of Science, Technology and Innovation

Maytee Martinez Dominguez

Delegación Territorial de Ciencia, Tecnología y Medio

Ambiente (CITMA). Pinar del Río, Cuba

maite@citma.vega.inf.cu

Isabel García Rodriguez

Centro de Estudios de Gestión de Ciencias e Innovación.

Instituto Superior de Tecnologias y Ciencias Aplicadas

(INSTEC). Ministerio de Educación Superior (MES),

Cuba

igarcia@instec.cu

Elena Figueroa Cabrera

Departamento Informática, Facultad de Ciencias Técnicas.

Universidad de Pinar del Río Hermanos Saiz Montes de

Oca, Cuba

elena@upr.edu.cu

Adriana Fernández Sánchez

Universidad de Pinar del Río Hermanos Saiz Montes de

Oca, Cuba

andry6595@nauta.cu

\section{Resumen:}

En Cuba el Sistema de Programas y Proyectos constituye la forma organizativa para la dirección de la actividad de Investigación, Desarrollo e Innovación. En la provincia de Pinar del Río, durante el ciclo de vida de los proyectos de ciencia, tecnología e innovación se evidencia la obtención de resultados de la Ciencia con alto valor agregado. Sin embargo, se constató en el proceso de gestión falta de sistematización de los componentes metodológicos de la actividad de propiedad intelectual. Ante esta situación, las autoras proponen un sistema informático que contribuye a la evaluación de los mismos desde una perspectiva de la Propiedad Intelectual en su proceso de gestión. Se destaca en la metodología utilizada el análisis documental y bibliográfico, los métodos histórico-lógico y analítico, encuestas, entrevistas, el cuestionario, el criterio de expertos y los métodos estadísticos. Los aportes de la investigación se fundamentan en la implementación de una herramienta analítica, para la toma de decisiones, que asegura la aplicación de las actividades inherentes a la Propiedad Intelectual en cada una de las fases que componen el ciclo de vida de los proyectos. Ello favorece la práctica de nuevas conductas, modos de planificar y decidir, de manera que se correspondan, integren y armonicen coherentemente con la Política Nacional de Ciencia, Tecnología e Innovación y de la Propiedad Intelectual. Palabras clave: gestión de la propiedad intelectual, herramienta de evaluación, proyectos de ciencia, tecnología e innovación.

Palabras clave: Gestión de la propiedad intelectual, Herramienta de evaluación, Proyectos de ciencia, Tecnología e innovación.

\section{Abstract:}

In Cuba the system of programs and projects constitute the organizational form for the management of Research, Development and Innovation activity. In Pinar del Rio province the achievement of scientific results with high value added are a reality during the life cycle of projects of science, technology and innovation. However, in the management process, lack of systematization of 
the methodological components of the intellectual property activity was tested. In the light of this situation, the authors propose an informational system that contributes to the evaluation of such components from an intellectual property perspective in its management process. It stands in the methodology used, documentary and bibliographical analysis, historical-logical and analytical methods, the surveys, interviews, the questionnaire, expert opinion and statistical methods. The contributions of the research are based on the implementation of an analytical tool, for decision-making, that assures the inherent activities to intellectual property in each of the phases that compose the life cycle of the projects. This favors the practice of new behaviors, ways of planning and deciding, so that they would correspond, integrate and harmonize coherently with the National Policy of Science, technology and innovation as well as the intellectual property.

KEYWORDS: Management of intellectual property, Evaluation tool, Projects of science, Technology and innovation.

\section{INTRODUCCIÓN}

El creciente desarrollo de la ciencia, tecnología e innovación hacen del desarrollo de una nueva generación de indicadores un reto de relevancia política y social sobre la necesidad de ajustar las políticas estatales a las realidades de la demanda social.

En este escenario de cambios dinámicos, el capital intelectual y su capacidad de generación de activos intangibles ha devenido en factor estratégico como fuente de ventajas competitivas para alcanzar un posicionamiento en el mercado e, incluso, para sobrevivir; sin embargo, el valor de estos activos disminuirá si pueden ser copiados o imitados por otros similares.

El amparo jurídico de los nuevos conocimientos, tecnologías, diseños estéticos y demás creaciones intangibles asociadas para preservarlas de la utilización gratuita, no autorizada, y garantizar un retorno adecuado de las inversiones que el país destina a estos fines, así como no infringir los derechos de Propiedad Intelectual (PI) y registros vigentes en Cuba y en el extranjero, son acciones que en el orden de la PI se establecen como objetivos del Sistema de Ciencia, Tecnología e Innovación en Cuba (SCTI), instituidos en el 2001 por el Ministerio de Ciencia, Tecnología y Medio Ambiente (CITMA) y referidos por García Capote et al. (2001), que tiene como mayor desafío lograr productos con alto valor agregado y nivel competitivo que ayuden a elevar de forma sostenible la calidad de vida de la población.

Es importante reconocer que, aunque el sistema de PI en el mundo responde fundamentalmente a los intereses de los países más desarrollados, la información que se genera por este resulta una valiosa herramienta del quehacer institucional en la sustentación de estrategias inteligentes, tal como lo expresan varios autores (Guzmán Sánchez, 1999; Santos Riveras, 2003; Toso, 2006; Roca Campañá, 2008; Fernández y Romeu, 2009). Por otra parte, la literatura refiere a la propiedad intelectual como una herramienta que permite a los Estados y a las empresas diferenciarse de sus semejantes, permitiéndoles ser más competitivos (Ballesteros García y Bulla de la Hoz, 2016).

En Cuba, las amenazas y retos que impone la nueva concepción de la Gestión del Conocimiento conllevan a una estrategia para un futuro inmediato, de la cual parte de la PI y su gestión, vinculada estrechamente a la investigación, el desarrollo, la tecnología, la innovación, la producción y el comercio, son una condición necesaria (García, 2006). Así mismo la gestión de proyectos a nivel territorial es un tema que adquiere progresivamente una mayor connotación, como herramienta de alto grado de aplicación dado su impacto en la sociedad actual (Guerra Betancourt, Pérez Campdesuñer y Fornet Hernández, 2014).

La organización de la PI en los sistemas institucionales constituye una garantía para la sociedad, así como el ordenamiento e implementación de la misma a través de la gestión de ciencia y tecnología, la protección y difusión de resultados de conocimiento y como alternativa en la cadena de valor a ciclo completo de la innovación (Torres Pombert, 2010; Contreras Villavicencio, Suárez Gutiérrez, Moreno Cruz y Correa Alvarez, 2017).

Las investigaciones realizadas en el marco del proceso de adopción de las normas y objetivos establecidos por ambos sistemas (SCTI y de la PI) en Cuba, específicamente en la provincia de Pinar del Río, han permitido evidenciar esas debilidades y abundar en los principales problemas que afectan el encargo de la PI, 
en el proceso de gestión de proyectos de ciencia, tecnología e innovación (Fornet Hernández, Morejón Borjas, Torres Santander y Guerra Betancourt, 2008; Martínez Domínguez, 2007; Morejón Borjas y Velázquez Zaldívar, 2011; Morejón Borjas, 2012), siendo las más significativas:

- Falta de cultura de temas de la PI.

- Productos y servicios sin marcas comerciales y poco alcance de protección.

- Inobservancia de las normas jurídicas vigentes.

- Pérdida de "Know How" por divulgación de resultados científicos susceptibles de protección.

- No se incluyen los gastos en los presupuestos para amparar los pagos en que se incurre al obtener y mantener un derecho de PI.

- No se estipulan cláusulas y/o disposiciones adecuadas y oportunas en materia de PI, para los convenios de colaboración económica, científico-técnica y proyectos internacionales que garantice la plena protección de los derechos.

- No se evalúa la conveniencia de proteger los resultados tecnológicos no solamente por patentes, sino también por otras modalidades de la Propiedad Industrial o por el Derecho de Autor.

- No se emplea la información de PI como política tecnológica, comercial y cultural.

- No se utiliza la modalidad de modelos de utilidad para la protección de las pequeñas innovaciones, perfeccionamientos y mejoras de las tecnologías existentes.

- Desacertadas transferencias de tecnologías.

- Insuficiente preparación de los recursos humanos en la temática de PI.

- Carencia de mecanismos encaminados a proporcionar protección legal a los resultados que tienen su salida a partir de actividades de Investigación, Desarrollo e Innovación ( $\mathrm{I}+\mathrm{D}+\mathrm{i})$ y que en su mayoría son gestionados a través de los programas y proyectos de ciencia, tecnología e innovación.

De acuerdo con el análisis anterior se deriva el problema científico siguiente: ¿Cómo contribuir al perfeccionamiento de la gestión de proyectos de ciencia, tecnología e innovación a partir de la evaluación de la PI en Pinar del Río?

El objeto de estudio de la investigación está determinado por el proceso de gestión de proyectos de ciencia, tecnología e innovación en Pinar del Río, que tiene en su contenido como campo de acción el proceso de evaluación de la PI de los proyectos de ciencia tecnología e innovación en Pinar del Río.

Por ello en la investigación se traza como objetivo:desarrollar una herramienta de evaluación de la PI para los proyectos de ciencia, tecnología e innovación de Pinar del Río.

Se establece como objetivo específico el que se relaciona a continuación: diseñar la herramienta que permita asegurar la ejecución de un conjunto de acciones de PI que son esenciales en el proceso de gestión de proyectos de ciencia, tecnología e innovación en Pinar del Río.

Se sustenta la siguiente hipótesis: el desarrollo de una herramienta de evaluación de la PI para los proyectos de ciencia, tecnología e innovación en Pinar del Río contribuirá a la toma de decisiones, al perfeccionamiento del sistema de programas y proyectos y a asegurar la ejecución de un conjunto de acciones de la PI que son esenciales en el proceso de gestión de los mismos.

\section{Metodología}

El proceso de investigación se desarrolla aplicando métodos de nivel teórico, empírico y estadístico, entre los que se encuentran:

Método histórico-lógico: se logró el estudio del desarrollo teórico, desde el punto de vista jurídico sobre los enfoques y tendencias en los temas de la PI y Sistema de Programas y Proyectos, que permitieron hacer las deducciones y reflexiones correspondientes sobre el objeto de estudio de la investigación. 
Método analítico: se analizó minuciosamente la legislación nacional e internacional, logrando una evaluación crítica de las distintas regulaciones que en torno al tema de investigación existen.

Análisis documental y bibliográfico: se incluyeron textos, publicaciones seriadas y no seriadas, reglamentos, resoluciones, decretos ley, documentos reguladores en políticas de ciencia, tecnología e innovación y de la PI, tesis y fichas de trabajos, todo lo cual permitió estudiar, en la bibliografía disponible en diferentes soportes, el objeto de estudio y sustentar criterios y valoraciones emitidas.

La encuesta, la entrevista a expertos y el cuestionario se aplicaron a la comunidad científica con el objetivo de obtener información teórica y práctica acerca del tema de investigación. El intercambio de experiencias y conocimientos prácticos con personas vinculadas al tema permitió ampliar y enriquecer la investigación con criterios actuales.

Criterio de expertos: se procesaron a través del método Delphy, grupo de expertos para la validación de los aportes fundamentales de la investigación y para obtener razonamientos en el proceso del diseño de la metodología. Se organiza un diálogo anónimo entre los expertos consultados individualmente mediante cuestionario, con vistas a obtener un consenso general o, al menos, los motivos de discrepancias; la confrontación de las opiniones se lleva a cabo mediante una serie de interrogantes sucesivas, entre cada una de las cuales la información obtenida sufre un procesamiento estadístico-matemático.

Métodos estadísticos: se aplicó la herramienta para el análisis y procesamiento de datos. Se propone Estadística inferencial para la comprobación de hipótesis, con análisis no probabilístico con una muestra intencional, que se apoyará en programas como el SPSS.

\section{RESULTADOS Y DISCUSIÓN}

Para el análisis realizado se determinó la muestra de 228 Proyectos Territoriales de Ciencia e Innovación, de los cuales se evaluaron 101 proyectos concluidos. La figura 1 muestra que, en la gestión de los proyectos evaluados, en 35 casos (34\%) se generaron resultados de alto valor agregado de la ciencia, susceptibles a la protección de la PI. Entre éstos prevalecen las multimedias y los softwares que tienen implícito marcas de servicio y de productos y las bases de datos por años que tributan a ramas y sectores priorizados. 
FIGURA 1.

Modalidades del comportamiento en la gestión de $\mathrm{I}+\mathrm{D}+\mathrm{i}$.

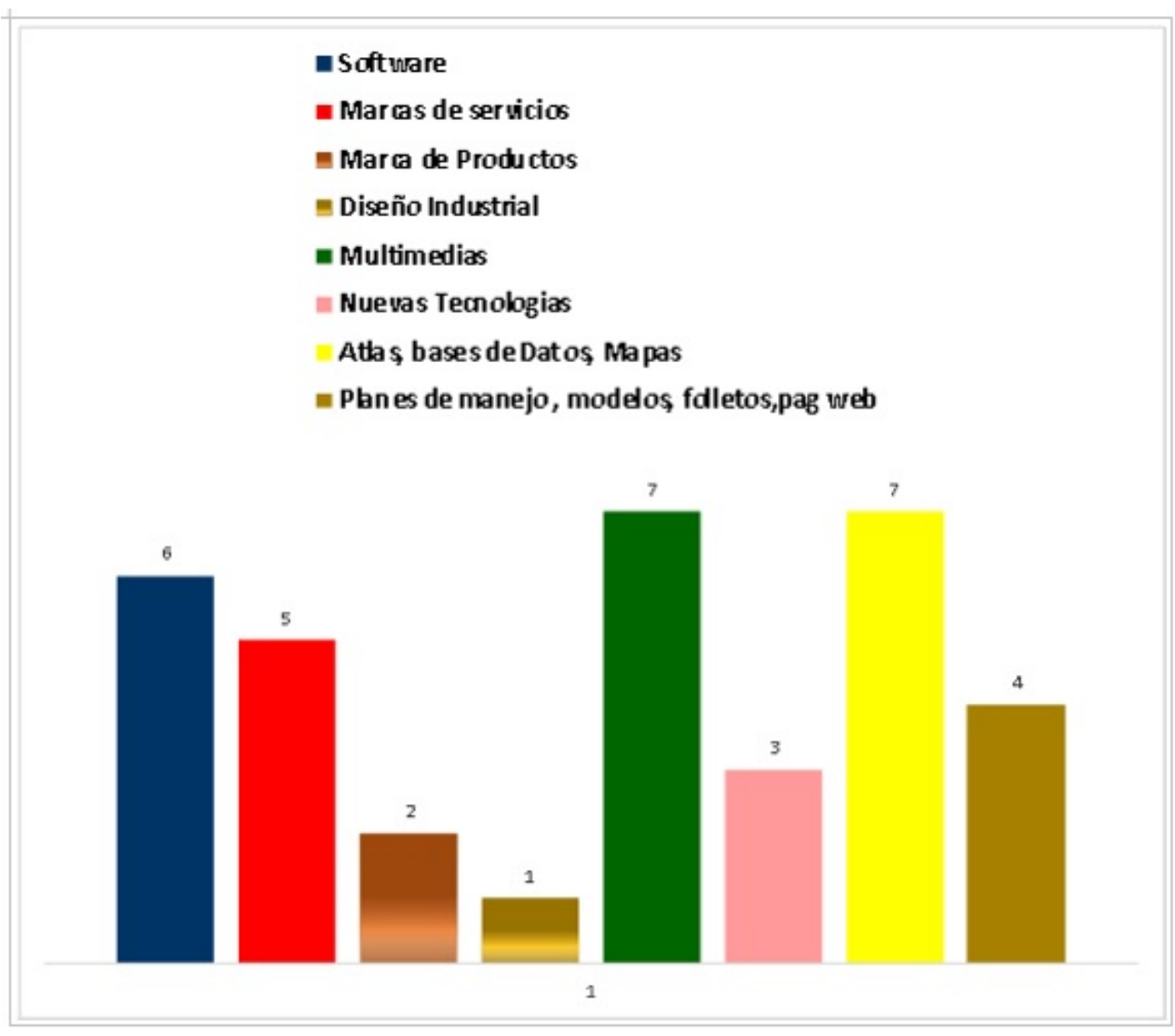

Fuente: Elaboración propia.

En el proceso de análisis, se examinaron los documentos incluidos en los expedientes de los 35 proyectos concluidos (perfil del proyecto, contrato, ficha de trabajo del evaluador, presupuesto, dictámenes de grupos de expertos, actas de compatibilización de la defensa, análisis costo-beneficio, informes de oponencias parciales y finales). En su totalidad no se incluyen las acciones relativas al tema de la PI, y no se inserta la amplia gama de actividades inherentes a ella para su adecuada gestión en los proyectos de ciencia, tecnología e innovación.

Se aplicó la encuesta a 97 investigadores en total, diez jefes de los PTCI, al 60\% de los miembros de los diferentes grupos de expertos y a 30 investigadores que ejercieron como jefes de los diferentes proyectos evaluados. Los resultados evidenciaron la falta de conocimiento en las actividades de la PI en la gestión de proyectos, carencia de instrumentos para la evaluación de los mismos, insuficiente gestión de la información de patentes en el proceso de $\mathrm{I}+\mathrm{D}+\mathrm{i}$ para la toma de decisiones, desconocimiento de los beneficios de proteger los resultados tecnológicos no solamente por patentes, sino por otras modalidades de la Propiedad Industrial o por el Derecho de Autor, así como de los servicios especializados en la temática (tabla 1). 
TABLA 1.

Resultados de encuesta aplicada a los actores de proyectos.

\begin{tabular}{|c|c|c|}
\hline OBJETIVOS & ENCUESTADOS & $\begin{array}{l}\% \text { de encuestados con conocimientos } \\
\text { sobre el objetivo evaluado }\end{array}$ \\
\hline $\begin{array}{l}\text { Niveles de la Cultura de propiedad } \\
\text { intelectual. }\end{array}$ & 97 & $30.4 \%$ \\
\hline $\begin{array}{l}\text { Formas de protección de los resultados } \\
\text { cientificos. }\end{array}$ & 97 & $12.37 \%$ \\
\hline $\begin{array}{l}\text { Consulta de la información de la } \\
\text { propiedad intelectual. }\end{array}$ & 97 & $6.19 \%$ \\
\hline $\begin{array}{l}\text { Propiedad Intelectual en la gestión } \\
\text { empresarial. }\end{array}$ & 97 & $8.25 \%$ \\
\hline Legislación. & 97 & $5.12 \%$ \\
\hline $\begin{array}{l}\text { Instrumento de Propiedad Intelectual para } \\
\text { evaluar los Proyectos de Ciencia, } \\
\text { Tecnologia e Innovación. }\end{array}$ & 58 & $0 \%$ \\
\hline $\begin{array}{l}\text { Inclusión de aspectos relacionados con la } \\
\text { propiedad intelectual en la gestión de } \\
\text { proyectos de ciencia, tecnologia e } \\
\text { innovación. }\end{array}$ & 97 & $5.1 \%$ \\
\hline
\end{tabular}

Fuente: Elaboración propia.

Se aplicó la entrevista al 50 \% de los Jefes de Programas Territoriales de Ciencia e Innovación (PTCI) y a los 18 miembros de mayor experiencia de los Grupos de Expertos de los PTCT con diez años de vigencia. El principal objetivo a medir se basó en el conocimiento de las actividades de la PI en la gestión de proyectos; fundamentalmente, en la valoración del instrumento utilizado para la evaluación de los mismos, se constató:

- Insuficiente dominio relacionado con la PI en la gestión de proyectos y ausencia de instrumentos metodológicos referativos para evaluar las disposiciones necesarias y adecuadas en materia de PI, que deben estar presentes en todo el ciclo de vida de los proyectos.

- Desconocimiento de PI y de la utilidad y beneficios económicos y sociales que reportaría de aplicarse, insuficiente cantidad de especialistas preparados para ejecutar y evaluar esta temática.

- Carencia de documentos que puedan constituir base material de estudio para orientar al sector empresarial y a la comunidad científica hacia este objetivo. Este es un elemento que incide desfavorablemente en los enfoques científicos que se debe conceder en la gestión de los proyectos de ciencia, tecnología e innovación.

Se señala que aun cuando las prioridades están sustentadas sobre la base de la obtención e introducción de nuevas y/o mejoradas variedades, tecnologías, introducción de logros científico-tecnológicos, desarrollo y/o asimilación de tecnologías de avanzada, no se prevé la utilización de la PI como una herramienta estratégica para la toma de decisiones, ni de protección de esos resultados generados en el proceso de gestión de proyectos de ciencia e innovación.

Otros datos importantes de estudios que precedieron refieren que, en el periodo 2005-2012, en Pinar del Río se declararon abandonados por la Oficina Cubana de la Propiedad Industrial 45 signos distintivos (uno de ellos asociado a una patente de invención). Las causas fundamentales fueron: no pago de concesión y 
no pago de renovación de los derechos adquiridos de la PI (Martinez Domínguez y Difurniao Grau, 2010; Difurniao, 2013).

En relación con el tema anterior, el $11 \%$ de los signos abandonados procedían como salidas y/o resultados de proyectos territoriales, evidenciando que no se previeron dentro de las fases del ciclo de vida del proyecto las actividades relacionadas con la planificación de los gastos para la protección legal y el mantenimiento de los derechos de PI adquiridos, así como para la tramitación de la protección legal de las tecnologías y marcas $\mathrm{u}$ otros signos distintivos ni para el pago de las tarifas de los servicios sobre PI que se requiera. Vale destacar que, según el clasificador internacional de productos y servicios, el 60\% de los signos distintivos abandonados tributaban a la nomenclatura de sustitución de importaciones y fondos exportables territorial.

En el caso de los registros de software, las autoras antes mencionadas refieren la no existencia de experiencias de licenciamiento o comercialización por parte de las instituciones del territorio. No se realiza un seguimiento a su utilización y mantenimiento, lo que rompe con la esencia de la figura del titular y sus derechos sobre las obras protegidas.

Compartiendo los criterios de Lis-Gutiérrez (2013), la combinación adecuada de instrumentos de gestión de PI requiere tener en cuenta el contexto jurídico e institucional, el perfil, objetivos y las capacidades de innovación de las propias organizaciones; además, la efectiva gestión de los activos intangibles en los proyectos de investigación adquieren una relevancia que comienza con la identificación de los mismos y su puesta en circulación como pilar fundamental en la trasferencia del conocimiento y el ciclo de las innovaciones (Unceta Satrústegui y Pomares Urbina, 2014).

A continuación, se exponen los elementos principales en los que se sustenta la Herramienta de evaluación de la PI para los proyectos de ciencia, tecnología e innovación en Pinar del Río.

\section{FUNDAMENTOS}

Los antecedentes de la Herramienta de evaluación de la PI para proyectos de ciencia, tecnología e innovación, se fundamentan sobre la base de una propuesta metodológica diseñada por Martinez Domínguez (2007) para la inclusión de la PI en los proyectos, como resultado de su tesis de maestría. La herramienta constituye un valor agregado a la metodología, que permite a los expertos que participan en el proceso de evaluación la realización de valoraciones en cuanto a la eficiencia y eficacia del desempeño de la PI en el quehacer de la actividad científico tecnológica agenciada por los proyectos y, así mismo, para que sean valorados desde una perspectiva de la PI en su proceso de gestión.

Las exigencias legales por las que se rige la herramienta propuesta son las establecidas por el Ministerio de Ciencia, Tecnología y Medio Ambiente (CITMA, 2002), regulados en la resolución N. ${ }^{\circ}$ 21/2002 que establece el Sistema Nacional de Propiedad Industrial, resolución N. ${ }^{\circ} 44 / 2012$, reglamento para el proceso de elaboración, aprobación, planificación, ejecución y control de los programas y proyectos de ciencia, tecnología e innovación (CITMA, 2012 y 2014). De la misma manera con lo legislado en el país en materia de Derecho de Autor y con la Actualización de los Lineamientos de la Política Económica y Social del Partido y la Revolución, aprobados en el VII Congreso del Partido Comunista de Cuba en el mes de abril de 2016, con especial referencia a los Lineamientos 5; 10; 14; 67; 68, 74; 80;83; 89; 92, 95; 99; 110; 111; 112 y 177, y la Política para el Sistema Nacional de la Propiedad Industrial en Cuba.

\section{Contribución de LA Herramienta}

La herramienta propuesta favorece la planificación y toma de decisiones, así como el desarrollo de estrategias tecnológicas y comerciales adecuadas, incrementa la capacidad innovadora y de desarrollo tecnológico, permite mayor involucramiento en la protección del patrimonio intelectual socialista, así como 
el perfeccionamiento del Sistema de Programas y Proyectos en su proceso de gestión. Por otro lado, mejora los estándares de calidad en el proceso de evaluación de la PI en los proyectos de ciencia, tecnología e innovación mediante el uso de las Tecnologías de la Informática y las Comunicaciones como parte del desarrollo de la automatización y de la política de informatización del país.

En la figura 2 se muestra la Herramienta metodológica a través de un sistema informático para la evaluación de la PI en los proyectos de ciencia, tecnología e innovación desarrollada con el lenguaje de programación JAVA (1.8 jdk), bien estructurado, orientado a objetos y permite que los programas sean ejecutados indistintamente en una gran variedad de equipos con diferentes microprocesadores y sistemas operativos (Bloch, 2001). Se utiliza el entorno de desarrollo integrado NetBeans (8.02) para escribir, compilar, depurar y ejecutar el programa.

Así mismo, esta herramienta posibilita el establecimiento de tres criterios fundamentales (como se muestra en las figuras 3, 4 y 5), los cuales por su incidencia en esta esfera de actividad permiten agrupar las acciones definidas en la metodología propuesta en estudio anterior (Martinez Domínguez, 2007), de manera que exista correspondencia entre la ficha del evaluador vigente y el método que se pretende introducir, desarrollando así una adecuada gestión de la PI en el tránsito paralelo al ciclo de vida de los proyectos de ciencia, tecnología e innovación, con incidencia en la mejora del Sistema de Programas y Proyectos a nivel territorial con el uso adecuado de las Tecnologías de la Informática y las Comunicaciones.

FIGURA 2.

Herramienta de evaluación de la PI para proyectos de $\mathrm{I}+\mathrm{D}+\mathrm{i}$.

\section{Ficha de Trabajo del Evaluador} Tema Propiedad Intelectual
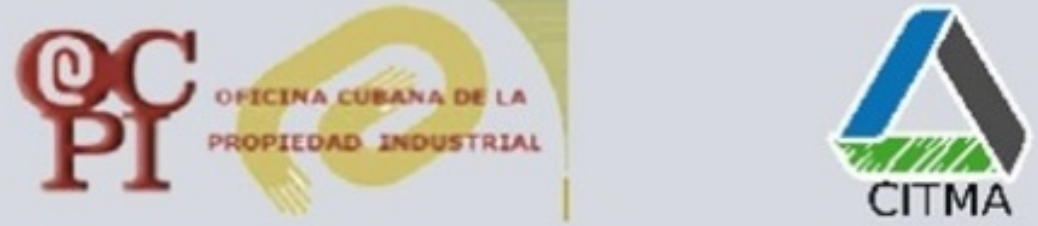

Criterios de Propiedad Intelectual:

Consulta de la Información Cientfíico Técnica

Protección del Resultado Obtenido

Introducción de los resultados

Evaluar Criterios 
La figura 3 muestra 13 criterios que responden a acciones encaminadas a la exploración del estado del arte, indicando acciones necesarias y oportunas (antecedentes, tendencias, estudios de mercado, enfoques contemporáneos, oportunidades competitivas, entre otros) que no deben dejar de contemplarse en la fase inicial de todo proyecto, facilitando la identificación de ideas o alternativas a ejecutar, propiciando el planteamiento del problema a resolver y los objetivos a alcanzar, caracterizándose el entorno, estimándose los recursos financieros, estudios del mercado potencial y elección de alternativas más viables para la gestación y gestión del nuevo conocimiento.

\section{FIGURA 3}

Criterio -1 Consulta de información científico-técnica

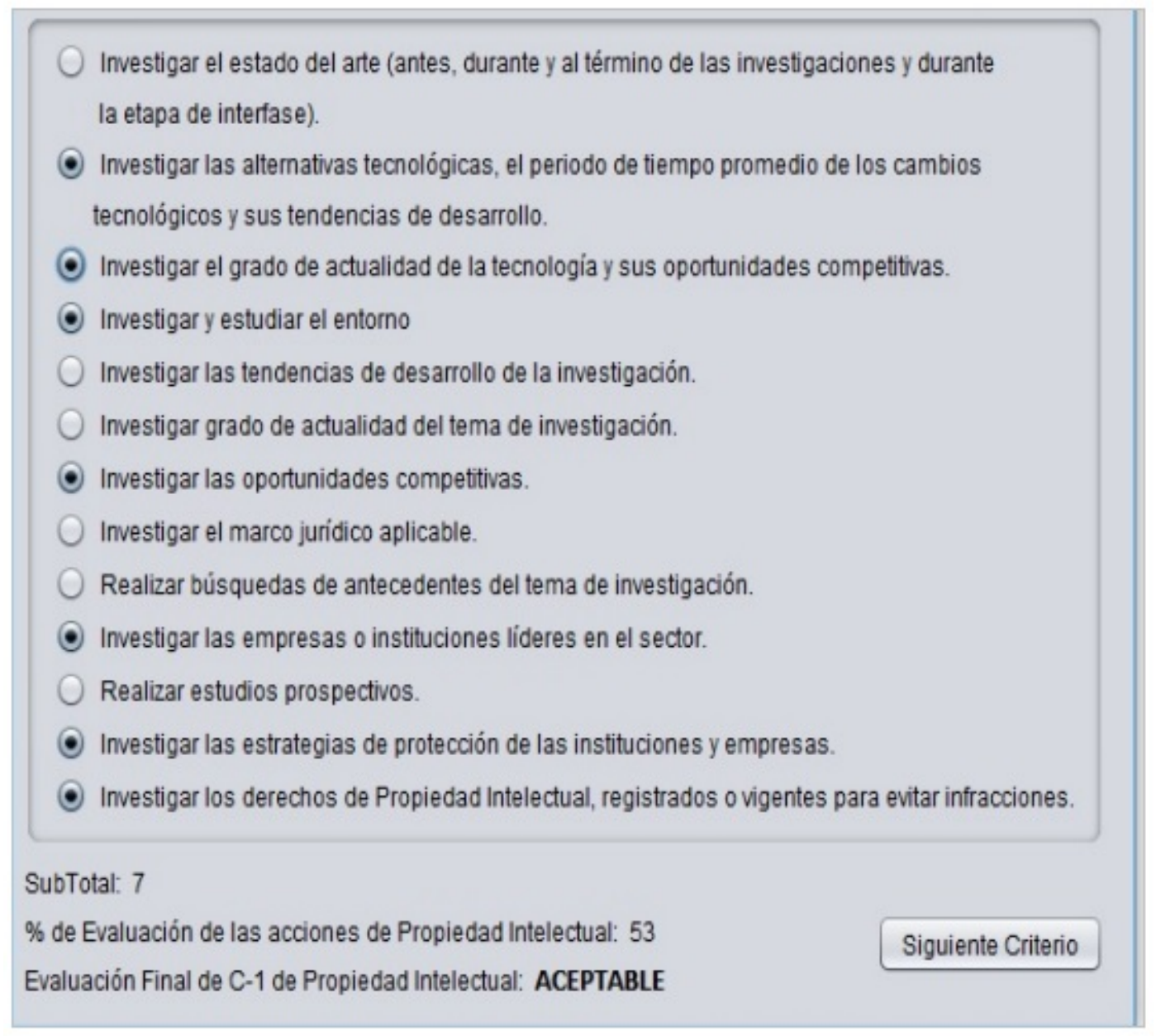

Fuente: elaboración propia

La figura 4 expone siete criterios basados fundamentalmente en la protección del nuevo conocimiento, unido a la identificación de los recursos humanos, materiales y financieros necesarios, análisis y la ejecución de la factibilidad técnico-económica que se requiera. 
FIGURA 4

Criterio-2 Protección del resultado obtenido.

Investigar si se debe dar curso a la investigación o procede la adquisición de una tecnología.

Investigar si la investigación resulta suficiente respecto al acervo de nuevos conocimientos.

Incorporar el presupuesto para los gastos de tramitación y protección legal de los derechos de propiedad intelectual y para el pago de las tarifas de los servicios que se requieran durante toda la investigación.

Determinar si el resultado obtenido satisface los requisitos exigidos para su protección así como establecer los medios posibles y procedimientos necesarios para la protección de la iinformación no divulgada con valor tecnológico y comercial.

- Determinar cobertura técnica que deberá ser reivindicada. (Patentes o modelos industriales).

- Evaluar la conveniencia de proteger en Cuba y en el extranjero. No obligatorio

Elaborar los documentos que conforman las solicitudes de protección legal.

SubTotal: 2

\% de Evaluación de las acciones de Propiedad Intelectual: 28

Evaluación Final de C-2 de Propiedad Intelectual: CUESTIONABLE

Siguiente Criterio

Fuente: elaboración propia

En la figura 5 se muestran los indicadores que marcan la puesta en marcha de la interface, con incidencia en el logro de la utilización de la PI en el diseño de estrategias para la toma de decisiones en los procesos de innovación y su comercialización; así también la capacitación del capital humano y, con ello, la ejecución de un conjunto de actividades encaminadas a la introducción de los resultados, como parte del cierre del ciclo de la investigación (alianzas estratégicas para la distribución comercial, asociaciones económicas, producciones cooperadas, venta del producto, concesión de licencias a terceros, entre otros). Así mismo comprende la transferencia de los resultados y el seguimiento de los mismos en el proceso de introducción o producción en el mercado. 
FIGURA 5.

Criterio-3 Introducción de los resultados.

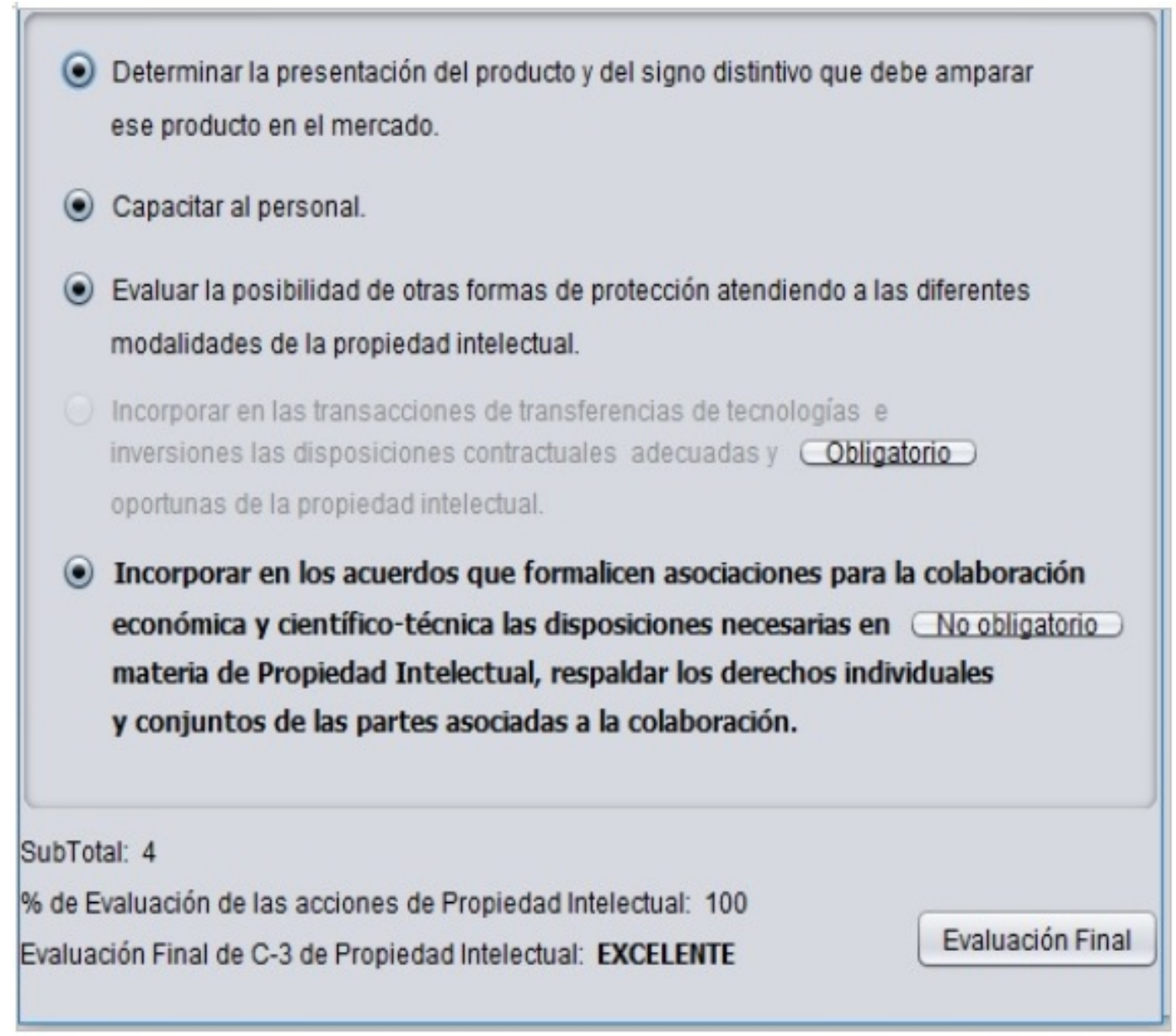

Fuente: elaboración propia

Por otra parte, las 30 acciones que se establecen en los criterios a evaluar por los expertos (figuras 3, 4 y 5) pueden asumir dos posibilidades, NO MARCAR cuando no se realizan, y MARCAR cuando se realizan. Las mismas agrupadas en su suma forman tres valores que su suma total pueden ser 15, 8 y 7 respectivamente; esto permite evaluar el porciento de cumplimiento de cada criterio de agrupación, tomando en cuenta el valor porcentual del criterio. Se evalúan a su vez de la siguiente forma:

1. Evaluación del criterio 1: a) mayor o igual a 80: Excelente, b) mayor o igual a 60: Bien, c) mayor o igual a 40: Aceptable, d) mayor o igual que 20: Cuestionable, e) menor o igual que 20: MAL.

2. Evaluación del criterio 2: a) igual a 100: Excelente, b) mayor o igual a 60: Bien, c) mayor o igual a 40: Aceptable, d) mayor o igual que 25: Cuestionable, e) menor o igual que 25: MAL.

3. Evaluación del criterio 3: a) igual a 100: Excelente, b) mayor o igual a 65: Bien, c) mayor o igual a 50: Aceptable, d) mayor o igual que 25: Cuestionable, e) menor o igual que 25: MAL.

Para llegar a la Evaluación Final de Proyecto en materia de Propiedad Intelectual se realiza a través de la combinación de los mismos:

C: Combinaciones existentes.

P: Cantidad de posibles respuestas en la evaluación del criterio n. 
$C=P_{1} \cdot P_{2} \cdot P_{3}$

$\mathrm{P} 1=\mathrm{P} 2=\mathrm{P} 3=5$

Por tanto, obtenemos: $\mathrm{C}=53$ obteniendo un total de 125 combinaciones.

Cada una de estas combinaciones posee una evaluación final que de forma similar puede ser Excelente, Bien, Aceptable, Cuestionable o Mal. La evaluación se realiza de forma automatizada después de haber agrupado las combinaciones con criterios condicionales, de forma tal que se obtenga el resultado razonablemente esperado. La herramienta por sí misma emite una evaluación final y se pueden evaluar las acciones que se dejaron de ejecutar, reflejándolas en las recomendaciones que se pueden derivar.

Las autoras de la presente investigación coinciden con los autores reportados en considerar importante la gestión de la PI y sus potencialidades en las organizaciones. Consideran que permite desarrollar tecnologías propias y de calidad, capaces de competir en los mercados internacionales, así como su papel trascendental en el desarrollo de políticas sociales y económicas para contribuir al bienestar de toda la sociedad, generadora de valor estratégico, que aprovecha los beneficios de la creatividad, el ingenio y la capacidad innovadora, propicia la obtención de resultados con potencial de uso, suscita los desafíos y oportunidades a las que los gestores de la investigación y los políticos se enfrentan, entre otros. En concordancia con los trabajos de Acuña, Schmal y Klein (2011), Morejón Borjas (2012) y Difurniao (2013) reflexionan, además, en que la transformación de la cultura del conocimiento en innovación, ciencia y tecnología tiene mucho que ver con la gestión adecuada y oportuna de las actividades de la PI derivada de la investigación científica y tecnológica. En ese sentido, el tema es aún insuficiente y débil, debido a barreras en el orden fundamentalmente del conocimiento y de su no utilización como herramienta de gestión de los SCTI y de la PI.

\section{Conclusiones}

- A partir del análisis de los fundamentos teóricos-metodológicos, así como de los resultados del estudio del comportamiento del SCTI en Pinar del Río, se evidencia un conjunto de deficiencias y de efectos negativos derivados de la ausencia de procedimientos y herramientas metodológicas que gestionen la PI en cada una las fases que componen el ciclo de vida del proyecto.

- La Herramienta de evaluación de la PI para proyectos de ciencia, tecnología e innovación permite a los expertos que participan en el proceso de evaluación la realización de valoraciones en cuanto a la eficiencia y eficacia del desempeño de la PI en el quehacer de la actividad científico tecnológica agenciada por los programas de ciencia, tecnología e innovación, así mismo, para que sean valorados desde una perspectiva de la PI en su proceso de gestión.

- Asimismo, la herramienta mejora los estándares de calidad en el proceso de evaluación de la PI en los proyectos de ciencia, tecnología e innovación mediante el uso de las Tecnologías de la Informática y las Comunicaciones como contribución de valor agregado a la metodología existente.

\section{Fuentes}

Decreto-Ley No. 14/1977. Derecho de Autor. Sobre la protección por Derecho de Autor a las obras científicas, artísticas, literarias y educacionales de carácter original. Gaceta Oficial Ordinaria (9), 30 de diciembre de 1977. Cuba. Recuperado de http://www.gacetaoficial.cu/html/leyderechoautor.htm 


\section{REFERENCIAS}

Acuña, D., Schmal, R. y Klein, P. (2011). Una Plataforma Web para Gestionar los Derechos de Propiedad Intelectual. Journal of Technology Management \& Innovation, 6(4), 258-275. Recuperado de http://www.jotmi.org/index. $\mathrm{php} / \mathrm{GT} /$ article/view/cas39/667

Ballesteros García, S. y Bulla de la Hoz, J. (2016). Incidencia de la propiedad intelectual en el desarrollo nacional y empresarial en el contexto de globalización actual. Revista La Propiedad Inmaterial, 22, 5-18. http://dx.doi.or $\mathrm{g} / 10.18601 / 16571959 . \mathrm{n} 22.01$

Bloch, J. (2001). Effective Java: Programming Language Guide. Addison Wesley

Contreras Villavicencio, D. M., Suárez Gutiérrez, E., Moreno Cruz, M. M. y Correa Alvarez, P. (2017). Propiedad industrial para la gestión de Ciencia, Tecnología e Innovación en empresas estatales cubanas. Revista La Propiedad Inmaterial, 23, 147-169. https://doi.org/10.18601/16571959.n23.06

Difurniao, G. H. (2013). Propuesta de Manual para la Gestión de la Propiedad Intelectual en la Universidad de Pinar del Rio Hermanos Saiz Montes de Oca (Tesis de maestría), Oficina Cubana de la Propiedad Industrial, La Habana, Cuba.

Fernández, H. A. y Romeu, L. E. (2009). Indicadores de patentes para sustentar la toma de decisiones estratégicas en los proyectos de I+D+i. Revista Rendija, 10. Recuperado de http://www.ocpi.cu

Fornet Hernández, E., Morejón Borjas, M., Torres Santander, M. E. y Guerra Betancourt, K. (2008). Resultados de la conducción del proceso de gestión de innovación a escala territorial: provincia de Holguin, Cuba. En O. Galante y A. Arcienaga (comp.), XII Seminario Latino-Iberoamericano de Gestión Tecnológica, ALTEC (pp. 347-357). Buenos Aires.

García Capote, E. et al. (2001). Fundamentos de la ciencia y la innovación tecnológica. Documento digital, Instituto Superior de Tecnologías y Ciencias Aplicadas, La Habana, Cuba.

García, B. (2006). La Propiedad Intelectual en la ciencia y el desarrollo en las universidades. Gestión de Ciencia e Innovación Tecnológica en las Universidades. La Habana: Félix Varela.

Guerra Betancourt, K., Pérez Campdesuñer, R. y Fornet Hernández, E. (2014). Propuesta de una tecnología para la gestión de proyectos de innovación en el sistema territorial de ciencia e innovación en Cuba. Revista Cubana de Información en Ciencias de la Salud, 25(4), 367-381. Recuperado de http://www.acimed.sld.cu/index.php/aci $\mathrm{med} /$ article/view/595/428

Guzmán Sánchez, M. V. (1999). Patentometría: herramienta para el análisis de oportunidades tecnológicas (Tesis de maestría), Universidad de Murcia, España; Universidad de La Habana, Cuba; Universidad Autónoma de México, México.

Lis-Gutiérrez,J. P. (2013). Gestión de la Propiedad Intelectual en las organizaciones. Una revisión de la literatura reciente. Recuperado de https://mpra.ub.uni-muenchen.de/47757

Martinez Domínguez, M. (2007). Metodología para la inclusión de la propiedad intelectual en el proceso de gestión de proyectos de ciencia e innovación (Tesis de maestría), Oficina Cubana de la Propiedad Industrial, La Habana, Cuba.

Martinez Domínguez, M. y Difurniao Grau, H. (2010). Propuesta de base de datos de signos distintivos como fuente de información comercial para la toma de decisiones. Avances, 12(4). Recuperado de http://ciget.pinar.cu/Rev ista/No.2010-4/articulo.htm

Ministerio de Ciencia Tecnología y Medio Ambiente [CITMA]. (2014). Indicaciones Metodológicas para la actividad de Programas y Proyectos de Ciencia, Tecnología e Innovación. La Habana, Cuba.

Ministerio de Ciencia, Tecnología y Medio Ambiente [CITMA]. (2001). Documentos rectores de la ciencia y la innovación tecnológica en Cuba. La Habana, Cuba.

Ministerio de Ciencia, Tecnología y Medio Ambiente [CITMA]. (2002). Resolución No.21/2002 que establece el Sistema Nacional de Propiedad Industrial. Gaceta Oficial de la República de Cuba. Recuperado de http://www gacetaoficial.cu 
Ministerio de Ciencia, Tecnología y Medio Ambiente [CITMA]. (2012). Resolución No.44/12. Gaceta Oficial de la República de Cuba. Edición Ordinaria, 11, 28 de marzo de 2012. Recuperado de http://www.gacetaoficial.cu

Morejón Borjas, M. M. (2012). Tecnología para la gestión de la propiedad intelectual en la empresa estatal cubana. Aplicación en organizaciones empresariales de la provincia Holguin. (Tesis doctoral), Instituto Superior de Tecnologías y Ciencias Aplicadas, La Habana, Cuba.

Morejón Borjas, M. M. y Velázquez Zaldívar, R. (2011). Propuesta de una tecnología para la gestión de la propiedad intelectual en la empresa estatal cubana. $A C I M E D, 22(3), 204-218$. Recuperado de http://scielo.sld.cu/pdf/ac i/v22n3/aci03311.pdf

Roca Campañá, A. (2008). La innovación tecnológica y la gestión de la propiedad intelectual. Ponencia presentada en III Congreso de Propiedad Industrial, abril, La Habana, Cuba.

Santos Riveras, A. (2003). La propiedad intelectual en el Sistema de Ciencia e Innovación Tecnológica. Ponencia presentada en Encuentro Latinoamericano de Patentes, ELDIPAT, 25 al 28 marzo, La Habana, Cuba.

Torres Pombert, A. (2010). Diseño de sistemas internos de propiedad intelectual en el modelo cubano: una propuesta metodológica. ACIMED, 21(1), 44-56. Recuperado de http://scielo.sld.cu/pdf/aci/v21n1/aci05110.pdf

Toso, F. (2006). La propiedad intelectual como herramienta de desarrollo económico. Ponencia presentada en Seminario Internacional "La propiedad intelectual como herramienta de gestión en los sistemas nacionales de ciencia, tecnología e innovación", 24, 25 y 26 de abril, La Habana, Cuba

Unceta Satrústegui, A. y Pomares Urbina, E. (2014). Innovación y propiedad intelectual. Antecedentes. Normas y Transformaciones en el marco comunitario. Argumentos de razón técnica 17, 137-154. Recuperado de http://i nstitucional.us.es/revistas/argumentos/17/art_8.pdf 